\title{
De la surprise du Dasein
}

Conjectures sur deux phrases de Sein und Zeit

\section{Claude Romano}

\section{(2) OpenEdition}

\section{Journals}

Édition électronique

URL : http://journals.openedition.org/alter/421

DOI : $10.4000 /$ alter.421

ISSN : 2558-7927

Éditeur :

Association ALTER, Archives Husserl (CNRS-UMR 8547)

\section{Édition imprimée}

Date de publication : 1 décembre 2016

Pagination : 47-68

ISBN : 978-2-9550449-2-6

ISSN : 1249-8947

Référence électronique

Claude Romano, « De la surprise du Dasein », Alter [En ligne], 24 | 2016, mis en ligne le 01 décembre 2017, consulté le 20 avril 2019. URL : http://journals.openedition.org/alter/421 ; DOI : 10.4000/ alter.421 


\section{DE LA SURPRISE DU DASEIN CONJECTURES SUR DEUX PHRASES DE SEIN UND ZEIT}

Claude Romano

Le moins que l'on puisse dire est que la question de la surprise (Überraschung) ne paraît pas revêtir un statut central dans le chef d'œuvre philosophique de Heidegger, puisque ce mot même y est absent et qu'on n'y découvre, en tout et pour tout, que deux uniques occurrences de deux de ses dérivés : Überraschtwerden [être surpris] et Überraschende [surprenant], présents dans deux phrases de la page 355. Le passage où ils interviennent est le suivant:

Umgekehrt gründet die Möglichkeit des Überraschtwerdens durch etwas darin, daß das gewärtigende Gegenwärtigen eines Zuhandenen ungewärtig ist eines anderen, das in einem möglichen Bewandtniszusammenhang mit jenem steht. Das Ungewärtigen des verlorenen Gegenwärtigens erschließt allererst den "horizontalen« Spielraum, innerhalb dessen Überraschendes das Dasein überfallen kann.

La traduction d'Emmanuel Martineau donne :

Inversement, la possibilité de l'être-surpris par quelque chose se fonde en ceci que le présentifier attentif d'un à-portée-de-la-main est inattentif à un autre à-portée-de-la-main se tenant avec le premier dans un contexte possible de tournure. C'est l'in-attention propre au présentifier perdu qui ouvre pour la première fois l'espace de jeu «horizontal » à l'intérieur duquel du surprenant peut assaillir le Dasein.

Pour comprendre le statut que reçoit ici la surprise, il convient tout $\mathrm{d}$ 'abord de réinscrire les deux phrases mentionnées dans le contexte d'ensemble de l'analyse de la temporalité en tant que sens du souci, c'est-à-dire en tant que sens de l'être même du Dasein. À la fin du 
$\S 65$, Heidegger énumère quatre thèses fondamentales permettant de circonscrire le concept extatique de temporalité (Zeitlichkeit) tel qu'il ressort des analyses de la seconde section de Sein und Zeit : 1) «Le temps est originairement comme temporalisation de la temporalité en tant que laquelle il possibilise la constitution de la structure du souci $»^{1}$. En $\mathrm{d}^{\prime}$ autres termes, le temps « $\mathrm{n}^{\prime \prime}$ est" absolument pas un étant»; il "n'est pas, mais se temporalise $»^{2}$; il ne se produit ou $\mathrm{n}^{\prime}$ advient en tant que tel qu'en conformité avec des modes d'existence du Dasein, et plus précisément avec la manière dont celui-ci se rapporte à son pouvoir-être le plus propre, s'élance anticipativement en direction de sa mort pour exister sur le mode de la résolution (Entschlossenheit), assumant par là la finitude de ses possibles (temporalité authentique) ou, au contraire, la manière dont il esquive la finitude essentielle de son être et succombe à l'inauthenticité ; 2) «La temporalité est essentiellement extatique $»^{3}$. Par là, il faut entendre que le temps n'est pas d'abord un étant subsistant (vorhanden) qui serait pourvu en outre de la caractéristique de sortir de lui-même, il est « le "hors-de-soi" originaire en et pour soi-même »", le hors-de-soi pur et simple (schlechtin). Ainsi, chacune des extases qui constituent la temporalité est à comprendre comme une modalité du transport extatique du Dasein vers... ce à quoi il se rapporte, et la temporalité se temporalise à chaque fois dans la cohésion et l'unité indissoluble de ses trois extases: l'avenir (Zukunft) entendu comme modalité de l'advenue à soi du Dasein à partir de son pouvoir-être le plus propre, l'avoir-été (Gewesenheit) qui articule la manière dont le Dasein « hérite de lui-même » et se rapporte à sa facticité, et le présent, et notamment cette modalité authentique du présent que Heidegger désigne sous le nom d' «instant (Augenblick)»; 3) «La temporalité se temporalise originairement à partir de l'avenir $»^{5}$. Cela est vrai en réalité uniquement de la temporalité authentique dans laquelle le Dasein se rapporte anticipativement à ses possibles finis et, à partir de là, fait retour vers son être-en-dette le plus propre pour l'assumer et en décider dans l'instant de la résolution. Inversement, la temporalité inauthentique, comme on va le voir, se temporalise à partir de l'extase du présent,

\footnotetext{
${ }^{1}$ M. Heidegger, Sein und Zeit, Tübingen, Niemeyer, 16e éd., 1986, p. 331 ; traduction d'E. Martineau, Etre et temps, Paris, Authentica, 1985, p. 232.

${ }^{2}$ Ibid. p. 328 ; trad. citée, p. 231.

${ }^{3}$ Ibid., p. 331 ; trad. citée, p. 232.

${ }^{4} \mathrm{Ibid} .$, p. 329 ; trad. citée, p. 231 ; voir aussi M. Heidegger, Grundprobleme der Phänomenologie, GA 24, p. 378 ; traduction de J.-F. Courtine, Les Problèmes fondamentaux de la phénoménologie, Paris, Gallimard, 1985, p. 321.

${ }^{5}$ M. Heidegger, Sein und Zeit, op. cit., p. 331 ; trad. citée, p. 232.
} 
c'est-à-dire du simple présentifier (Gegenwärtigen). 4) «Le temps originaire est fini $»^{6}$. « Le temps» originaire renvoie ici à la temporalisation authentique de la temporalité, laquelle, comme on l'a vu, se confond avec l'assomption résolue par le Dasein de sa propre mortalité sur l'arrière-plan de laquelle seulement prennent sens tout choix d'un destin singulier, toute décision factice en faveur de telle ou telle possibilité d'existence, et donc aussi toute possibilité de répéter telle ou telle possibilité d'être héritée ou choisie. La temporalité n'est pas seulement finie, elle est ce qui infuse notre être même de finitude ; elle constitue, à cet égard, le sens même de l'être du Dasein et même le sens de l'être en tant que tel.

La temporalité authentique se caractérise par conséquent par l'unité d'une anticipation résolue de la mort (extase de l'avenir), d'une répétition (Wiederholung) par laquelle le Dasein se rapporte à chaque fois à l'extase de son avoir-été à la lumière de son pouvoirêtre le plus propre, et d'une décision résolue en faveur de telle ou telle possibilité d'existence - résolution (Entschlossenheit) qui seule ouvre la dimension de l'instant (Augenblick), c'est-à-dire de la modalité authentique de l'extase du présent. Cet Augen-blick doit être entendu littéralement dans son sens de «clin d'œil », ou plutôt de « coup d'œil » jeté sur une situation - coup d'œil grâce auquel cette situation acquiert pour la première fois son relief et se détache en tant que telle. Seul, en effet, le Dasein prêt à la mort et résolu peut accéder par là même à l'instant dans lequel sa situation lui devient visible en son entièreté :

Le présent, tel qu'il est re-tenu dans la décision résolue et en résulte, c'est ce que nous nommons l'instant (Augenblick) [...] Dans l'instant au sens d'ekstase, le Dasein qui existe résolument est transporté (entrückt) dans des possibles à chaque fois déterminés facticiellement - les circonstances, les péripéties de la situation où s'exerce son action. L'instant (Augenblick) est ce qui, en provenance de la décisionrésolue, peut seul et en premier lieu, d'un coup d'œil, apprécier en quoi consiste la situation d'agir. L'instant est cette modalité insigne de l'existence résolue dans laquelle le Dasein, à titre d'être-au-monde, appréhende d'un coup d'œil son monde? .

L'instant est donc toujours «synoptique » : il abrite en lui la possibilité d'une syn-opsis en vertu de laquelle le Dasein, en tant que résolu, est transporté devant lui-même et la possibilité de se choisir comme tel et en totalité, c'est-à-dire aussi d'atteindre à une parfaite cohésion

${ }^{6}$ Idem.

7 M. Heidegger, GA 24, p. 407-408; trad. citée, p. 345. 
de son existence à l'encontre de ce qui apparaîtra, par contraste, comme la dispersion de l'existence journalière et inauthentique, de l'existence se déroulant sous l'emprise du On.

Cette caractérisation très générale de la temporalité extatique du Dasein en tant que temporalité authentique permet d'envisager ce qui constitue l'objet propre du $\S 69$ (a), à savoir l'autre manière dont cette temporalité peut se temporaliser, sa manière déchue ou inauthentique. Pour pouvoir décrire ce phénomène, il est nécessaire de repartir du mode d'être dans lequel le Dasein se tient « de prime abord et le plus souvent»- mode d'être neutre à l'égard de la différence de l'existence authentique et inauthentique : le mode d'être de la quotidienneté (Alltäglichkeit). Il est vrai que Heidegger va par la suite rectifier en partie ce jugement et soutenir que la quotidienneté du Dasein se ramène en dernière analyse à un mode d'existence inauthentique ${ }^{8}$. Quoi qu'il en soit - car ce point n'est pas décisif pour la question qui nous occupe - Heidegger affirme ici que, dans la quotidienneté caractérisée par l'être-dans-la-moyenne, le Dasein se laisse absorber par ses tâches et se comprend lui-même à l'aune de l'étant dont il se préoccupe. Loin de s'appréhender lui-même comme un étant qui, en son être, existe, c'est-à-dire est essentiellement pouvoir-être, projet en direction de ses possibles - et donc aussi comme un étant qui, parce qu'il est mortel, ne peut se rapporter à ses possibles que comme essentiellement transis de finitude - le Dasein s'appréhende au contraire à partir des outils qu'il manie et des tâches qu'il se propose d'accomplir, c'est-à-dire de l'étant qui possède le mode d'être de la Zuhandenheit, de l'être-disponible ou de l'être-à-portée-de-la-main. La temporalité de la quotidienneté reçoit de là sa configuration spécifique. «Tout à ses tâches » le Dasein quotidien se rapporte à l'extase de l'avenir non sur le mode du devancement et de l'anticipation résolue, mais sur celui de l'être-tendu vers... (Gewärtigen) telle ou telle tâche à accomplir, telle ou telle «affaire » qui sollicite son attention. Cette être-tendu et attentif prescrit son sens à l'avenir inauthentique. Il diffère de la simple attente (Erwartung), puisque cette dernière puise au contraire en lui sa possibilité : "L'être-tendu vers (Gewärtingen) n'est donc pas une variété de l'attente, mais à l'inverse, $l^{\prime}$ attente trouve son fondement dans l'être-tendu $»^{9}$. Cet être-tendu vers ce dont il retourne à chaque fois avec la préoccupation, c'est-àdire vers l'étant disponible auquel le Dasein se rapporte, est indis-

\footnotetext{
8 Voir, par exemple, M. Heidegger, Sein und Zeit, op. cit., p. 178 ; trad. citée (modifiée), p. 139 où Heidegger parle de « la quotidienneté inauthentique (uneigentlichen Alltäglichkeit)».

${ }_{9}^{9}$ M. Heidegger, GA 24, p. 410-411 ; trad. citée (modifiée), p. 348.
} 
sociable d'un conserver ou d'un retenir (Behalten) qui constituent la modalité de transport du Dasein en direction de ce qui vient juste de se produire, de ce qui n'a pas fini de l'occuper et de l'accaparer. Mais en étant ainsi tendu vers l'affaire en cours et en retenant ce qu'il vient juste d'effectuer, ce qui l'occupait un moment plus tôt, le Dasein se perd de vue lui-même et "s'oublie » en un sens spécifique, il fuit son avoir-été authentique et se le dissimule: "L'extase constitutive de l'oubli se caractérise comme une fuite devant l'avoir-été le plus propre, de telle sorte que celle-ci occulte cela même devant quoi il y a fuite $»^{10}$. La modification inauthentique de l'avoir-été conduit ainsi à une dégradation de celui-ci en simple passé (Vergangenheit) que le Dasein n'a plus à prendre en charge en propre à la lumière de son pouvoir-être, dont il n'a plus à décider, mais qu'il lui suffit de retenir et de conserver en mémoire en s'oubliant lui-même. Enfin, dans la temporalisation inauthentique de la temporalité comme tension vers... qui retient et conserve (en détournant le Dasein de lui-même), règne aussi, pour ce qui touche à la troisième extase, non plus cette modalité authentique du présent qu'est l'instant (Augenblick), mais le simple présentifier (Gegenwärtigen) grâce auquel le Dasein se rapporte à l'affaire en cours, à ce qu'il convient de faire et est opportun dans le moment même. La temporalité de la préoccupation quotidienne peut ainsi être déterminée comme un s'attendre qui se temporalise en conjonction avec un retenir oublieux et un présentifier. Elle est un "présentifier qui s'at-tend et retient (gewärtigend-behaltende Gegenwärtigen) ${ }^{11}$, ou encore « un être-tendu oublieux-présentifiant $»^{12}$. Cette temporalité inauthentique est une manière, pour le Dasein, d'esquiver la nécessité de son choix existentiel de lui-même, une soustraction au Dasein de son propre choix, par contraste avec laquelle la résolution peut être définie comme une re-saisie ou une réappropriation de ce choix, un « choix du choix (Wählen der Wahl) » ${ }^{13}$ permettant au Dasein de se « gagner » lui-même et de se posséder luimême en s'arrachant à l'irrésolution du On.

De cette temporalité de la préoccupation procède ce que Heidegger appelle «temps du monde (Welt-zeit)». En effet, dans sa quotidienneté affairée, le Dasein «a » ou $\mathrm{n}^{\prime}$ «a » pas le temps, il compte avec le temps et décompte le temps en fonction des affaires dont il se préoccupe. Ce temps du monde répond à trois caractéristiques prin-

\footnotetext{
${ }^{10}$ M. Heidegger, GA 24, p. 411 ; trad. citée, p. 348.

${ }^{11}$ M. Heidegger, Sein und Zeit, op. cit., p. 354 ; trad. citée (modifiée), p. 246.

12 M. Heidegger, GA 24, p. 412 ; trad. citée (modifiée), p. 349.

${ }^{13}$ M. Heidegger, Sein und Zeit, op. cit., p. 268 ; trad. citée, p. 195.
} 
cipales : 1) databilité ; 2) écartement ; 3) publicité. Tout d'abord, il est essentiellement datable, car il est ressaisi expressément en tant que tel à travers l'usage quotidien des horloges : le Dasein préoccupé ne peut compter avec le temps que parce qu'il compte le temps, le soumet à un constant calcul, ce qui entraîne d'ailleurs que, généralement il " n'a pas le temps", et ce qu'il décompte ainsi ce sont des maintenant(s) dans leur relation avec le moment présent. Le temps du monde est par conséquent aussi un «temps du maintenant $»^{14}$, un temps où le futur est un simple «maintenant » qui n'est pas encore et le passé un "maintenant» qui n'est plus. En second lieu, le temps ainsi mesuré est toujours d'une «étendue » variable : il se caractérise par son écartement, il s'étend de tel moment à tel autre, il s'étire parfois en longueur dans l'ennui. Enfin, ce temps est essentiellement " public », comme les horloges qui permettent de le mesurer : il est le temps banal, commun, indifférent, il est le temps de tout le monde et de personne. C'est sur la base de ces différentes caractéristiques que se produit le nivellement du temps du monde aboutissant à ce que Heidegger appelle le «concept vulgaire de temps »-qui constitue aussi le concept de temps qui règne d'un bout à l'autre de l'histoire de la pensée occidentale, d'Aristote à Hegel et au-delà - et dans lequel le temps est fondamentalement compris comme « une suite de maintenant flottant en l'air, qui se trouve là simplement et qu'il suffit de reconnaître dans son être-donné ${ }^{15}$. Ce nivellement ôte au temps du monde sa databilité, son écartement et même sa publicité pour le ramener à un unique continuum unidimensionnel dans lequel, à chaque instant, un maintenant succède à un autre maintenant, ces « maintenant(s) » étant conçus selon le mode d'être de la simple présence subsistante (Vorhandenheit). Le temps vulgaire est une succession indéfinie de maintenant(s), un temps infini dans les deux sens, sans commencement ni fin.

\section{Le statut de la surprise}

Nous avons maintenant au moins un aperçu schématique du contexte général dans lequel prennent place les affirmations de Sein und Zeit relatives à la surprise. En effet, dans le maniement circonspect de l'étant à portée de la main qui rapporte celui-ci à ce dont il retourne avec lui, à sa «tournure »(Bewandtnis) ou à sa finalité,

\footnotetext{
${ }^{14}$ Ibid., p. 421 ; trad. citée, p. 287.

${ }^{15}$ M. Heidegger, GA 24, p. 379 ; trad. citée, p. 323.
} 
c'est-à-dire à son renvoi constitutif à d'autres outils à l'intérieur d'un complexe instrumental, peuvent se présenter deux situations qui " dérangent» le commerce absorbé du Dasein avec ses outils et ses affaires: 1'une est celle où un outil vient à faire défaut et se révèle in-disponible, inemployable, c'est-à-dire non-à-portée-de-la-main, donnant lieu à ce que Heidegger appelle au $\S 69$ (a) le «ne-plusretrouver, [le] manquer de (Vermissen) »; la seconde est celle où, au contraire, le Dasein trouve devant soi en lieu et place de ce vers quoi il était tendu quelque chose à quoi il ne s'attendait pas, quelque chose qui contrarie sa préoccupation absorbée, en sorte qu'il est pris de court et cède à la surprise (Überraschung). La surprise est donc d'emblée caractérisée par l'excès de ce qui se trouve à portée-de-main sur l'être-tendu vers (Gewärtigen) en tant que ce dernier rend possible toute présentification (Gegenwärtigung) de quelque chose : le surprenant est ce qui vient déjouer l'attente et l'attention - incluses toutes deux dans le concept de Gewärtigen -, ce qui, ce faisant, déstabilise radicalement le Dasein et le prend de court, mais qui ne peut le déstabiliser ainsi que parce que ce qui se présente à lui est ici fondamentalement in-attendu (Un-gewärtigen), c'est-à-dire précédé d'aucune attente et attention, et donc nullement maîtrisé ou jugulé dans son caractère dérangeant ou menaçant par aucune présentification préalable. En somme, la surprise paraît relever d'une déficience ou d'un déficit de la tension-vers du Dasein, de son attente-attention exprimée par le terme "Gewärtigen », permettant ainsi à la chose d'échapper à sa prise et de glisser dans l'in-attendu, de se dérober au jeu réglé de l'attention prévenante et de la présentification avisée et circonspecte.

La surprise trahit ainsi sa proximité fondamentale avec la peur mais non point avec l'angoisse - comme l'indique en passant un autre passage de Sein und Zeit. La surprise (ou tout au moins l'être-intrigué qui est une variété de surprise en mode mineur: Stutzigwerden) y figure, en effet, au nombre des modifications de la peur: "Nous connaissons encore, écrit Heidegger, d'autres modifications de la peur sous les noms de timidité, de réserve, d'anxiété, de surprise (Stutzigwerden). En tant que possibilités du se-trouver (affection), toutes ces modifications renvoient au fait que le Dasein comme être-aumonde est "intimidé" »16. Ce rapprochement de la surprise avec la peur est hautement significatif. En effet, en vertu de toutes les grandes distinctions qui structurent $\hat{E}$ tre et temps (peur/angoisse, authenticité/inauthenticité, fuite devant soi-même/être soi-même authenti-

16 M. Heidegger, Sein und Zeit, op. cit., p. 142 ; trad. citée p. 118. 
que, aliénation/liberté, etc.), il ne semble pas possible d'envisager quelque chose comme une surprise authentique (et une temporalisation authentique de la temporalité laissant place à la surprise) -, tout comme il ne semble pas y avoir dans Sein und Zeit de place pour un événement (Ereignis) qui arriverait au Dasein et pourrait ressortir à autre chose qu'une compréhension inauthentique de lui-même. En effet, et aussi étrange que cela puisse paraître - nous avons tenté de le montrer jadis ${ }^{17}$ - chaque fois que l'événement (Ereignis, Geschehnis, Vorkommnis) intervient de manière centrale et conceptuellement significative dans l'économie de l'ontologie fondamentale, il est mis par Heidegger au compte de l'inauthenticité. Citons pour mémoire l'analyse de l'être-pour la mort dans laquelle c'est le On, et lui seul, qui comprend le mourir (Sterben) comme simple décès (Ableben), c'est-àdire comme "un événement bien connu, survenant à l'intérieur $\mathrm{du}$ monde (bekanntes innerweltlich vorkommendes Ereignis) »18 et, afin d'échapper au vertige de l'angoisse, "prend soin d'inverser cette angoisse en une peur d'un événement (Ereignis) qui arrive ${ }^{19}$; ou encore, les analyses de l'appel de la conscience et de l'historialité du Dasein dans lesquelles c'est à nouveau le On qui comprend l'appel de la conscience comme procurant des informations sur "des événements du monde (Weltereignisse) ${ }^{20}$, et c'est encore lui qui comprend à tort l'historialité (Geschichtlichkeit) du Dasein à partir de "l'homme comme "sujet" des événements (Ereignisse) ${ }^{21}$. Ainsi, non seulement l'événement apparaît toujours du côté de l'impropre dans Sein und Zeit mais, plus profondément, en le caractérisant par l'Innerweltlichkeit, en lui attribuant le mode d'être de la Wirklichkeit par opposition à l'existence, en le ravalant dans la sphère de l'impropre, Heidegger finit par priver l'événement de tout statut existential, ou, ce qui revient au même, dépouille l'existence de toute dimension événementielle - ou événementiale. Nous n'y reviendrons pas ici.

Si seul un Dasein déchu et inauthentique, s'oubliant lui-même dans ses tâches et existant loin de lui-même, semble être sujet à la surprise, inversement, le Dasein résolu et authentique qui a atteint, de ce fait même, à une pleine cohésion de son existence sous l'horizon de la mort, semble être affranchi de la surprise - si du moins on cherche à interpréter le silence de l'ouvrage sur ce point. N'est-il pas vrai, en effet, que le Dasein qui s'est résolu et s'est emparé dans un choix

\footnotetext{
${ }_{17} \mathrm{Cl}$. Romano, « Le possible et l'événement », in Il y a, Paris, PUF, 2003, p. 55-111.

${ }^{18}$ Ibid., p. 253 ; trad. citée, p. 186.

${ }^{19} \mathrm{Ibid} .$, p. 254 ; trad. citée, p. 187.

${ }^{20}$ Ibid., p. 273 ; trad. citée, p. 198.

${ }^{21}$ Ibid., p. 379 ; trad. citée, p. 262.
} 
fondateur de son existence et l'a embrassée du regard dans le coup d'œil de l'instant ne peut plus être dépassé par rien - y compris sans doute par ce qui vient déjouer ses attentes? "Lorsque la résolution, écrit Heidegger, en devançant, a repris la possibilité de la mort dans son pouvoir-être, l'existence authentique du Dasein ne peut plus être dépassée par rien ${ }^{22}$. Cette affirmation ne vaut-elle pas aussi et d'abord du surprenant, c'est-à-dire aussi de ce qui est susceptible de faire succomber le Dasein à la peur? Certes, Heidegger rappelle qu'il ne faut pas comprendre l'authenticité comme un " état d'exception (Ausnahmezustand) $»^{23}$ définitivement affranchi de toute rechute dans l'inauthenticité, mais seulement comme une modification existentielle de cette dernière. Même le Dasein résolu continuera à jouer son rôle sur le théâtre de la quotidienneté, il ne sera jamais entièrement affranchi ni de la peur ni de la surprise. Il n'en reste pas moins que le statut de la surprise pose question - par son ravalement au statut d'existential inauthentique. N'y a-t-il donc de surprise pour le Dasein qu'en régime de déchéance? La temporalité authentique doit-elle être pensée principiellement comme soustraite à toute surprise?

Nous voudrions aborder ces questions - qui restent probablement sans réponse dans l'Hauptwerk heideggérien - en faisant l'hypothèse de deux sources bien différentes pour une telle idée, deux sources qui, par leur opposition même, délimitent quelque chose comme la position originale (et à certains égards instable) de Sein und Zeit : une source chrétienne et une source stoïcienne. D'un côté la conception paulinienne de l'instant (kairos) et plus généralement du temps eschatologique, mais dépouillée de toute référence à une parousie; de l'autre, le présent stoïcien vécu tout entier par le sage dans la conscience de l'imminence de la mort et enclos dans des limites étroites, par opposition au temps dispersif et indéfini de l'existence de l'insensé.

\section{Le premier modèle : le modèle paulinien}

Nous trouvons en effet très tôt dans l'itinéraire de Heidegger, dès le cours de 1920-21, Introduction à la vie religieuse, dans le commentaire minutieux qu'il consacre à la première épître de Paul aux Thessaloniciens, et plus particulièrement dans l'interprétation phénoménologique qu'il avance de la notion d'instant (kairos/Augenblick), des

\footnotetext{
${ }^{22}$ M. Heidegger, Sein und Zeit, op. cit. p. 307 ; trad. citée, p. 219.

${ }^{23} \mathrm{Ibid}$., p. 130 ; trad. citée, p. 110.
} 
éléments susceptibles d'éclairer fortement cette relégation au second plan de la surprise. Ce qui intéresse tout particulièrement Heidegger dans ce cours est la manière dont l'apôtre présente à ses "frères » la relation qu'il convient d'entretenir à la parousia du Seigneur, c'est-àdire à "la nouvelle manifestation du Messie qui s'est déjà manifesté ${ }^{24}$. À leur question pleine d'inquiétude : «Quand cette parousie se produira-t-elle? ?, Paul ne répond pas en indiquant un moment, ni $\mathrm{d}^{\prime}$ ailleurs en indiquant qu'il ignore ce moment; rien de ce qu'il dit ne se situe sur le plan de la simple connaissance, car c'est d'un tout autre rapport à cette parousie qu'il y retourne avec la foi et avec l'espérance, un rapport appelé à transformer entièrement la vie du chrétien ici et maintenant. Heidegger insiste tout particulièrement sur la substitution d'une question à une autre qui déplace entièrement le problème du terrain de la connaissance à celui de la vie : ce n'est plus la question du «quand? » (quand se produira l'événement du retour du Christ en gloire), mais celle du «comment?» (comment dois-je transformer ma vie ici et maintenant dans l'espérance de ce retour ?) qui devient ici rectrice pour la vie du fidèle: "Paul élabore sa réponse, affirme Heidegger, en opposant deux façons de vivre [...]. Ce qui est décisif, c'est la manière dont je me rapporte à cet événement dans la vie authentique (eigentlichen Leben). Cela définit le sens $\mathrm{du}$ "quand", du temps et de l'instant (Augenblick) »25. Le temps et l'instant de la parousie ne se définissent donc plus en référence à un temps calendaire, au «temps du monde» tel que le décomptent les horloges, ni à l'attente (Erwartung) d'un simple événement à venir, mais à partir de la modalité d'accomplissement de la vie facticielle elle-même. Le passage de l'apôtre auquel se rapporte directement ce commentaire est le suivant :

Quant aux temps (khronoi) et aux instants (kairoi), vous n'avez pas besoin, frères, qu'on vous écrive là-dessus. Car vous-mêmes vous savez exactement que le jour du Seigneur vient comme le voleur la nuit. Quand ils diront: paix et sécurité, alors la perdition sera soudain sur eux comme les douleurs sur la femme enceinte, et ils n'échapperont pas. Mais vous, frères, vous n'êtes pas dans les ténèbres pour que le jour vous surprenne comme un voleur: car vous êtes tous fils de la lumière et fils du jour. Nous ne sommes pas

\footnotetext{
${ }^{24}$ M. Heidegger, Phänomenologie des religiosen Lebens, GA 60, p. 102 ; traduction de J. Greisch, Phénoménologie de la vie religieuse, Paris, Gallimard, 2012, p. 114.

${ }^{25}$ M. Heidegger, GA 60, p. 99-100 ; trad. citée, p. 111.
} 
de la nuit ni des ténèbres; ne dormons donc pas comme les autres ; au contraire, tenons-nous éveillés et sobres ${ }^{26}$.

L'opposition entre les deux manières de vivre, les deux modalités d'accomplissement de la vie facticielle auxquelles faisait référence Heidegger se reflète dans l'antithèse entre une requête de sécurité qui ne peut être satisfaite ("quand ils diront: paix et sécurité... ») et l'attitude d'éveil préconisée par l'apôtre. Ceux qui exigent paix et sécurité trahissent de cette manière leur attachement à ce monde, ils manifestent qu'ils vivent tout entiers dans l'amour de ce monde et se sont détournés de la relation authentique au Messie. Ils se bornent, en effet, à attendre un événement cosmique qui n'est pas encore présent mais le deviendra peut-être un jour. Tout autre est l'attitude de la seconde catégorie de fidèles, ceux qui ne vivent pas dans les ténèbres mais dans une attitude d'espérance (elpis) :

On pourrait penser de prime abord, écrit Heidegger, que l'attitude envers la parousia est une attente, et l'espérance chrétienne (elpis) est un cas particulier de celle-ci. Mais cela est totalement faux! [...] La structure de l'espérance chrétienne [...] diffère radicalement de toute attente. «Le quand et l'instant » [...] posent un problème particulier d'explicitation. Le «quand» pris au sens de l'optique particulière $\mathrm{d}^{\prime}$ un temps « objectif » n'est déjà plus pris originellement ${ }^{27}$.

Non seulement ceux qui se rapportent à la parousie sur le mode de l'attente, parce que leur préoccupation est tout entière pour ce monde-ci, parce qu'ils vivent dans les ténèbres à l'égard d'euxmêmes et de Dieu, ne se rapportent pas comme il convient à l'eskhaton de la fin des temps, mais en outre il se trouveront pris radicalement au dépourvu par le retour du Messie en gloire, qui les surprendra comme un voleur la nuit. Ici s'introduit la question de la surprise qui, comme on le voit, ne caractérise que la relation inauthentique à la parousie et la temporalité de la vie facticielle qui en découle. C'est le caractère subit de la perdition qui s'abat sur cette première catégorie de fidèles et leur surprise qui manifestent à quel point ils vivent dans les ténèbres. La perdition, commente Heidegger,

les prend au dépourvu, ils ne s'y attendent pas. Ou plutôt: ils se trouvent justement dans une attente conforme à une optique particulière ; leur attente est absorbée par tout ce que la vie leur apporte. Parce qu'ils vivent dans cette attente, la perdition les atteint de telle

${ }^{26}$ Première Épître aux Thessaloniciens, V, 1-6 ; nous citons les épîtres de Paul d'après la traduction de J. Grosjean et M. Léturmy, La Bible, Nouveau Testament, Paris, Gallimard, « La Pléiade », 1971. ${ }_{27}$ M. Heidegger, GA 60, p. 102 ; trad. citée (modifiée), p. 114. 
manière qu'ils ne peuvent pas s'enfuir. Ils ne peuvent se sauver euxmêmes parce qu'ils ne se possèdent pas eux-mêmes, parce qu'ils ont oublié leur propre soi ; parce qu'ils ne se possèdent pas eux-mêmes dans la clarté du savoir authentique ${ }^{28}$.

Le parallélisme entre l'attitude ici décrite par Heidegger et celle du Dasein immergé dans la quotidienneté et tourné tout entier vers les tâches de ce monde saute aux yeux : dans un cas comme dans l'autre, c'est précisément parce que le Dasein se laisse absorber par le monde qui le préoccupe qu'il se laisse aussi prendre à défaut et surprendre. Du reste, la connexion entre l'attente tournée vers le monde et les exigences de la quotidienneté est établie par Heidegger lui-même dans ce même passage : "Ceux qui disent "paix et sécurité" $(5,3)$ s'adonnent à ce que la vie leur apporte, ils s'occupent des tâches quelconques de la vie. Ils sont prisonniers de ce que la vie leur offre; ils sont dans les ténèbres en ce qui concerne le savoir que l'on a de soimême $»^{29}$. La surprise - exprimée dans l'épître de Paul par le verbe katalambanô, saisir : "pour que le jour vous surprenne/saisisse comme un voleur (hina hê êmera humas ôs kleptês katalabê) »- est donc ce qui prend à revers une attente tout entière tournée vers le monde et qui est aussi, de ce fait même, une forme d'oubli de soi.

Il en va tout autrement de la seconde catégorie de fidèles, les fidèles "authentiques", ceux qui ne se laissent pas absorber par le monde mais vivent tout entiers tournés vers la lumière, dans l'attitude de la foi véritable. Ceux-là ne demandent en aucune façon à être rassurés, ils vivent au contraire dans une insécurité totale, dans ce que Heidegger baptisera plus tard, en adoptant le vocabulaire de Kierkegaard, l'angoisse - et, seulement dans cette mesure, il sont replacés face à eux-mêmes et "se possèdent eux-mêmes » : "Pour la vie chrétienne, il $n^{\prime} y$ a pas de sécurité ; l'insécurité constante est également l'aspect caractéristique des significativités fondamentales (Grundbedeutenheiten) de la vie facticielle ${ }^{30}$. Ces fidèles ne se rapportent donc pas à l'eskhaton de la fin des temps comme à un événement à venir, qui se produira «bientôt» ou "plus tard", mais, en tout cas, pas maintenant; ils transforment leur vie dès à présent dans l'instant. Leur attitude est celle d'une veille dégrisée et sobre qui contraste avec le sommeil des premiers. Ils ne se laissent donc pas surprendre par la Seigneur parce que leur existence est déjà tout entière tournée vers lui et que, pour eux, l'eskhaton se produit en un

\footnotetext{
${ }^{28}$ M. Heidegger, GA 60, p. 103 ; trad. citée, p. 115.

${ }^{29}$ M. Heidegger, GA 60, p. 105 ; trad. citée, p. 117 (nous soulignons).

${ }^{30}$ Idem.
} 
sens à chaque instant par la transformation même de leur vie déjà advenue.

Il n'est pas utile d'insister davantage sur ce que le déclassement de la surprise dans Sein und Zeit peut devoir à l'épître de Paul. Cette épître (et son commentaire) semble d'ailleurs avoir joué un rôle de premier plan dans la genèse de la conception de la temporalité extatique tout entière : à l'être-pour-la-parousie du chrétien se substituera, dans l'ouvrage de 1927, l'être-pour-la-mort authentique ; à la «fin » qui n'a pas à être attendue comme un simple événement à venir mais doit transformer la vie entière dans l'instant correspondra le phénomène de la résolution, $c^{\prime}$ est-à-dire le devancement authentique de la mort en tant que retour vers l'être-en-dette le plus propre ouvrant conjointement la possibilité de la décision dans l'instant ; à la recherche obstinée de paix et de sécurité correspondra le "rassurement (Beruhigung)» qui est le signe distinctif de la quotidienneté moyenne ou médiocre et accompagne "l'affairement multiple de la préoccupation $»^{31}$; à la veille du fidèle véritable correspondra l'éveil du Dasein pour son pouvoir-être le plus propre ${ }^{32}$. Au ravalement du temps calendaire et objectif dans l'inauthentique la dérivation du «temps du monde » à partir de la temporalité authentique originaire ${ }^{33}$.

\section{Le second modèle : le modèle stoïcien}

Mais une seconde source peut être invoquée susceptible d'expliquer le statut de la surprise dans l'ontologie fondamentale: une source stoïcienne. Cette source est beaucoup moins obvie, puisque les traces d'une lecture du corpus stoïcien sont beaucoup plus ténues dans l'œuvre de Heidegger. Il est tout de même peu vraisemblable que l'auteur de Sein und Zeit n'ait pas eu connaissance d'auteurs

\footnotetext{
${ }^{31}$ M. Heidegger, Sein und Zeit, op. cit., p. 195 ; trad. citée, p. 149.

32 On pourrait étudier les reliquats de cette thématique de l'être éveillé (wach sein) dans l'ontologie fondamentale, notamment dans l'analyse de l'appel de la conscience (M. Heidegger, Sein und Zeit, op. cit., p. 275 ; voir aussi p. 286). Il est aussi question de manière assez significative de la façon dont la temporalité authentique «éveille » le présent authentique - l'instant : «La temporalité originaire et authentique se temporalise à partir de l'avenir authentique, et cela de telle manière que, étant-été de manière avenante, elle éveille (weckt) pour la première fois le présent » (M. Heidegger, Sein und Zeit, op. cit., p. 329 ; trad. citée p. 231). Le thème de la sobriété se retrouve lui aussi dans $\hat{E}$ tre et temps, notamment dans l'affirmation selon laquelle la résolution devançante « jaillit de la compréhension sobre (nüchternen) des possibilités fondamentales factices du Dasein " (M. Heidegger, Sein und Zeit, op. cit., p. 210). L'angoisse, elle aussi, est caractérisée comme «sobre (nüchternen)» (idem.).

33 Voir aussi Cl. Romano, «Le possible et l'événement », art. cit.
} 
comme Epictète, Marc-Aurèle ou Sénèque. L'influence d'un stoïcisme « diffus » sur la conceptualité de l'ontologie fondamentale a d'ailleurs été maintes fois relevée, par exemple par Paul Ricœur ${ }^{34}$. Nous avons là, en tout cas, un hypertexte bien davantage caché, ou du moins nonthématisé, sur lequel il est possible de se livrer à quelques conjectures. Heidegger souligne d'ailleurs lui-même que la notion de souci (Sorge) à laquelle il a recours a des sources non seulement néotestamentaires, mais également stoïciennes. La 220 fable de Hygin qu'il cite $^{35}$ dans Être et temps pour illustrer la notion ontologico-existentiale de Sorge, affirme-t-il dans les Prolegomena zur Geschichte des Zeitbegriffs, renvoie non seulement au terme merimna dans la Septante (sollicitudo dans la Vulgate), mais à l'usage courant de ce terme dans la philosophie stoïcienne - et Heidegger fait référence ici à la $90^{\mathrm{e}}$ lettre de Sénèque ${ }^{36}$. Toute la problématique de la possession de soi (en un sens bien sûr différent de celui auquel faisait allusion Heidegger à propos de l'épître de Paul) et de l'auto-appartenance du Dasein se tient, qui plus est, à l'arrière-plan du concept même d'Eigentlichkeit, de propriété ou d'authenticité, définie comme un être à soi-même, comme un se-posséder en propre. À propos de ce terme, Heidegger apporte en effet la précision suivante : "Eigentlichkeit doit être entendu ici au sens littéral (im wörtlichen Sinne) de se-posséder-en-propreen-étant-auprès-de-soi-même (Bei-sich-selbst-sich-zu-eigen-haben) ${ }^{37}$. Aussi, la résolution (Entschlossenheit) qui accomplit cette authenticité et opère l'authentification de l'existence tout entière peut-elle être décrite comme une manière de s'emparer de l'existence qui élève cette dernière à une forme d'auto-transparence : la résolution devançante est « ce comprendre - consécutif à l'appel de la conscience qui libère pour la mort la possibilité de s'emparer de l'existence et de dissiper radicalement tout auto-recouvrement fugace ${ }^{38}$. Comme l'écrit Sénèque, inaestimabile bonum est suum fieri: "inestimable bien que d'arriver à s'appartenir ${ }^{39}$.

\footnotetext{
34 Paul Ricœur, Temps et récit 3, Paris, Seuil, « Points essais», 1991, p. 101 : « N'est-ce pas, en effet, dans une configuration éthique, très marquée par un certain stoïcisme, que la résolution face à la mort constitue l'épreuve suprême d'authenticité ? » ; voir aussi p. 195.

${ }^{35}$ M. Heidegger, Sein und Zeit, op. cit., p. 197-198.

${ }^{36}$ M. Heidegger, Prolegomena zur Geschichte des Zeitbegriffs, GA 20, p. 419-420 ; traduction d'Alain Boudot, Prolégomènes à l'histoire du concept de temps, Paris, Gallimard, 2006, p. 437.

${ }^{37}$ M. Heidegger, GA 20, p. 390 ; trad. citée, p. 408.

${ }^{38}$ M. Heidegger, Sein und Zeit, op. cit., p. 310 : sur cet idéal de transparence dans le stoïcisme, on pourra par exemple se reporter à Marc-Aurèle, Pensées pour moi-même, X, 1 (nous citerons désormais les Pensées dans la traduction d'Émile Bréhier revue par J. Pépin, in Les stoïciens, Paris, Gallimard, « La Pléiade », 1962).

39 Sénèque, Lettres à Lucilius, 75, 18.
} 
Or cette auto-appartenance met en jeu, dans le stoïcisme, un autre genre de veille que celui auquel se référait la première épître aux Thessaloniciens. Une part importante de l'askesis stoïcienne repose, en effet, sur un travail de l'attention (prosokhê) qui ne doit jamais se relâcher, doit être maintenue constamment en alerte, y compris dans le sommeil ${ }^{40}$, afin justement que le sage ne soit jamais pris de court par un événement inattendu et conserve une totale maîtrise sur ses représentations. Dès que la pensée se relâche, dès que la tension de l'âme faiblit, peut se produire un dérèglement passionnel sur lequel il devient par la suite impossible d'agir. L'âme ne peut plus reprendre le contrôle d'elle-même et du mouvement qu'elle a laissé se développer par négligence. C'est pourquoi, tout sommeil de l'âme, toute inconscience, toute inadvertance sont dangereuses et doivent être vigoureusement combattus : potius exercitandus e somno et vellicandus est animus : "plutôt, sortons du sommeil et harcelons notre âme", affirme Sénèque ${ }^{41}$. Un aspect fondamental de cet exercice de l'attention consiste, dans le stoïcisme, à avoir la mort sans cesse présente à l'esprit, à la « devancer » comme dirait Heidegger, pour vivre chaque instant et accomplir chaque action comme si c'étaient les derniers : "Accomplis chaque action de la vie comme si c'était la dernière, en te tenant éloigné de toute légèreté ", exhorte Marc-Aurèle ${ }^{42}$. Ce même refus de la légèreté se retrouve en toutes lettres dans Sein und Zeit: "Seul le devancement de la mort expulse toute possibilité arbitraire et "provisoire" : seul l'être libre pour la mort donne au Dasein son but pur et simple et rejette l'existence dans sa finitude. La finitude saisie de l'existence arrache à la multiplicité sans fin des possibilités immédiatement offertes de la complaisance, de la légèreté (Leichtnehmen), de la dérobade et transporte le Dasein dans la simplicité de son destin ${ }^{43}$. Cette multiplicité sans fin dont le Dasein doit se détourner au profit de la finitude et de la simplicité de son destin - enraciné dans une temporalité elle-même finie - n'est pas sans évoquer l'aiôn de la temporalité de l'insensé, auquel s'oppose le présent fini, clos, délimité, mais s'étendant de ce fait même au temps tout entier, du sage en tant que ce dernier fait de chaque moment le lieu de son initiative morale.

\footnotetext{
${ }^{40}$ Voir par exemple Epictète, Entretiens, IV, 12, 1-19 où il est recommandé de ne jamais relâcher son attention et d'étendre cette attention à la totalité de la vie en n'excluant même pas le sommeil.

${ }^{41}$ Sénèque, Lettres à Lucillius, 20, 13.

42 Marc-Aurèle, Pensées pour moi-même, II, 5 (trad. modifiée).

${ }^{43}$ M. Heidegger, Sein und Zeit, op. cit., p. 384 ; trad. citée, p. 265. Sur la « légèreté » du On, voir Sein und Zeit, op. cit., p. 128 ; trad. citée, p. 108.
} 
Cette attention au présent impossible à prendre en défaut et qui est l'apanage du sage entraîne, dans le stoïcisme, une relégation de la surprise au rang de simple passion devant être éradiquée. Parmi les quatre grands genres de passions, chagrin (lupê), crainte, (phobos), désir (epithumia), plaisir (hêdonê $)^{44}$, la surprise (asmenismos) se subordonne au plaisir, puisqu'elle est définie comme « le plaisir d'un bien inattendu $»^{45}$, ou encore $«$ le plaisir des choses inattendues $»^{46}$. À cette première surprise, positive, s'oppose une surprise en négatif, la mauvaise surprise qui est une variété de la peur et doit donc être, elle aussi, vaincue : c'est l'ekplêxis, étonnement produit par un coup, effroi, stupeur. Elle est définie comme «la peur provenant de la représentation d'un fait imprévu (explêxis de phobos ek phantasias asunêthous pragmatos) ${ }^{47}$, ou encore comme un "étonnement excessif $»^{48}$. Tantôt subordonnée à la peur et tantôt au plaisir, la surprise est dans les deux cas une passion à éliminer, et elle ne possède probablement pas de forme "bonne», on ne trouve pas à son sujet d'équivalent d'une «passion bonne (eupatheia)» telle que la joie. Ainsi, de même que seul le Dasein in-attentif (ungewärtigen) pouvait être pris de court et céder à la surprise, de même la surprise est toujours dans le stoïcisme la manifestation d'un défaut de tension (tonos) de l'âme et donc aussi d'un défaut de constance et de tenue - de vertu (arêtê), (nous allons revenir dans un instant sur ces notions).

Mais l'un des lieux les plus patents de la possible analogie dont nous essayons ici de tracer les contours est probablement la conception même de l'instant comme lieu de totalisation de l'existence qui soustrait le Dasein à toute perte - et à toute perte de temps contrastant avec la temporalité dispersive et indéfinie de la quotidienneté médiocre, et, a fortiori, avec le concept vulgaire de temps, infini de part et d'autre. En s'élançant anticipativement vers sa fin et en existant sur le mode de la résolution, le Dasein se soustrait du même coup à la dispersion de l'existence irrésolue, il se rassemble et s'unifie dans l'horizon de sa propre finitude, accédant ainsi à la cohésion d'un destin ; il cesse de «perdre son temps » et a désormais tout son temps :

\footnotetext{
${ }^{44}$ Diogène Laërce, Vie et opinions des philosophes, VII, 110.

${ }^{45}$ H. von Arnim, Stoicorum Veterum Fragmenta, 3 vol., Leipzig, Teubner, 1903-1905 (abrégé désormais SVF), III, 401.

${ }^{46}$ Ibid., III, 401 et 402.

${ }^{47}$ Ibid., III, 407.

48 Ibid., III 411 (2).
} 
L'ir-résolu se comprend à partir des événements et des accidents qui font encontre et se pressent alternativement dans ce type de présentifier. Se perdant dans ce dont il se préoccupe, ou plutôt dans ce à quoi il s'affaire l'ir-résolu y perd son temps. D'où son discours caractéristique: «je n'ai pas le temps». De même que l'existant inauthentique perd constamment du temps et n'en «a » jamais, de même la temporalité de l'existence authentique se caractérise-t-elle de façon privilégiée par le fait que, dans la résolution, elle ne perd jamais de temps et « a toujours le temps ». Car la temporalité de la résolution a, du point de vue de son présent, le caractère de l'instant ${ }^{49}$.

L'instant est donc cette modalité du présent qui est soustraite à toute "perte » et s'oppose au présentifier fugace du On qui clame constamment " ne pas avoir le temps », comme le Lapin d'Alice au pays des Merveilles, et ce, parce qu'il ne cesse de "perdre son temps » en l'investissant dans des tâches aussi multiples que futiles, en s'affairant sans relâche sur le theatrum mundi. Ce présent totalisant qui rassemble synoptiquement le temps en le soustrayant à toute perte rappelle fortement la conception stoïcienne. Le temps de l'insensé, affirment les stoïciens, est un temps qui cesse de se perdre de part et d'autre, en direction du passé et du futur, dans l'indéfini; il est un fleuve s'écoulant entre deux abîmes et engloutissant tout. Au regard de ce temps, quelle différence cela peut-il faire de mourir jeune ou de vivre vieux, puisque ces deux durées sont aussi infinitésimales l'une que l'autre au regard de l'infinité des cycles cosmiques: "Regarde en arrière le gouffre du temps, écrit Marc-Aurèle, et en avant un autre infini. En lui, quelle différence entre trois jours de vie, et le triple de la durée de vie de Nestor ? »50 Cet «abîme du temps infini $»^{51}$, comme l'appelle aussi Marc-Aurèle, est à la fois celui du temps cosmique, infiniment divisible et dans lequel le maintenant présent est dépourvu de toute durée, et celui du temps de l'insensé52 dont la déperdition est continuelle, parce qu'il laisse filer l'instant présent et néglige de faire de celui-ci le lieu d'une initiative morale, se projetant sans cesse vers le passé et l'avenir - qui tous deux n'existent pas - et existant ainsi constamment hors de lui-même, esclave de ses passions.

\footnotetext{
${ }^{49}$ M. Heidegger, Sein und Zeit, op. cit., p. 410 ; trad. citée, p. 307.

${ }^{50}$ Marc-Aurèle, Pensées, IV, 50. Voir aussi V, 24.

${ }^{51}$ Marc-Aurèle, Pensées, IV, 3.

${ }^{52}$ Contre l'interprétation de Victor Goldschmidt, Pierre Hadot a souligné l'importance de distinguer entre deux présents bien différents dans la doctrine stoïcienne, le pur maintenant infinitésimal du temps cosmique et le "présent épais » de la conscience, que l'insensé ne cesse de quitter pour se perdre dans le passé dans le futur et céder à l'agitation passionnelle. C'est ce présent «épais » que Marc-Aurèle exhorte à « amoindrir » pour pouvoir le supporter : voir P. Hadot, La citadelle intérieure, Paris, Fayard, 1992, p. 154.
} 
À ce temps illimité dont le présent échappe sans cesse s'oppose le présent circonscrit et délimité par l'exercice de l'attention et la maîtrise des représentations qui est propre au sage - présent qui, désormais, "tient ferme », à l'image de celui qui s'y tient fermement, et englobe en lui, en quelque sorte, la totalité du temps, car, constamment achevé en lui-même, il est tel que rien ne lui fait défaut. L'exercice de l'attention et la maîtrise des représentations aboutissent ainsi à une stabilisation du temps dans le présent: "Efface l'imagination. Arrête cette agitation. Dans le temps, fixe le présent. Sache ce qui t'arrive à toi ou à autrui $»^{53}$. Le sage circonscrit le moment présent en cessant de se préoccuper du futur et du passé et en s'enfermant image de la citadelle intérieure - dans le seul présent, c'est-à-dire dans le seul temps qui soit offert à son initiative et dont il puisse s'emparer et se rendre maître ; ce faisant, il étend aussi les limites du présent au temps entier, puisque un tel présent ne manque plus de rien, il ne prend plus place dans l'infini du temps cosmique, n'est plus borné par le passé et le futur, mais, étant parfaitement achevé à chaque instant, confine à l'éternité. Tel était déjà le sens de la maxime selon laquelle il faut accomplir chaque action comme si c'était la dernière : se conduire de la sorte, écrit Pierre Hadot, "c'est vivre l'instant présent avec une telle intensité et un tel amour que toute vie, en quelque sorte, y soit contenue et $\mathrm{s}^{\prime} \mathrm{y}$ achève ${ }^{54}$.

Ainsi, comme le souligne Victor Goldschmidt, il existe dans le stö̈cisme deux présents bien différents : le présent circonscrit et délimité du sage qui comprend en lui la totalité du temps et met un terme à la déperdition continuelle de celui-ci dans une existence ballottée par le flot des passions, et le présent des illusions et des passions, justement, fuyant d'un côté comme de l'autre et à jamais illimité :

On distingue dans les textes deux mouvements en sens opposés : le présent de nos illusions que nous voudrions étendre aux dimensions de la vie universelle, va être ramené à un instant infinitésimal où se dissout la vie cosmique tout entière; à l'inverse, le présent instantané, ressaisi et déterminé par l'initiative morale, va s'élargir jusqu'aux confins des périodes cosmiques, de manière à inclure celles-ci dans ses propres limites ${ }^{55}$.

Contraction du temps infini en un instant évanescent, s'il n'est pas circonscrit par le sage ; expansion d'un moment limité à la totalité du temps, si le sage réforme sa vie et met à profit l'instant présent pour

\footnotetext{
${ }_{53}$ Marc-Aurèle, Pensées, VII, 29.

${ }^{54}$ P. Hadot, La citadelle intérieure, op. cit., p. 152.

55 V. Goldschmidt, Le système stoïcien et l'idée de temps, Paris, Vrin, 2ème éd., 1977, p. 194.
} 
en faire le lieu de la vertu. C'est ce que Goldschmidt désigne d'un terme qui s'appliquerait tout aussi bien à l'Augenblick heideggérien : « l'instant totalisant $»^{56}$.

La décision de "délimiter l'instant présent», écrit-il, parvient à s'opposer efficacement au flux qui emporte tout, à le stabiliser; elle permet de "posséder incontinent tous les biens que tu souhaites d'atteindre par un détour » (à travers le temps étranger) et de composer la vie avec la plénitude du kathortôma qui ne demande rien à $«$ l'ère successive ${ }^{57}$.

C'est pourquoi cet instant totalisant peut-être comparé à ces activités parfaites qui atteignent à chaque instant leur fin (comme la contemplation ou la vision chez Aristote), contrairement aux activités qui n'atteignent leur terme qu'à un moment donné, telles la danse ou le théâtre : l'âme raisonnable, écrit Marc-Aurèle,

atteint sa fin propre, à quelque moment que survienne la fin de la vie. Il n'en va pas comme d'une danse ou d'une pièce de théâtre, dont l'action entière reste inachevée, si elles sont interrompues. En toute partie de la vie et à quelque moment qu'on la saisisse, l'âme accomplit son projet pleinement et sans déficience, et elle peut dire : « Je recueille ce qui m'appartient » [...] à quarante ans, si l'on a assez d'intelligence, on a fini de voir en quelque sorte tout ce qui a été et tout ce qui sera sous une forme semblable ${ }^{58}$.

Il est possible ainsi de dégager dans l'analyse stoïcienne du temps trois niveaux qui correspondent assez bien à la différence heideggérienne entre temps du monde (temps mesurable infini), temps inauthentique (temps dispersé dans l'infinité des tâches qui nous accaparent et nous rendent oublieux de nous-mêmes) et temps authentique (devancement résolu de la mort en tant qu'il rend possible la décision ferme et «tenue » dans l'instant synoptique), correspondant respectivement au temps cosmique infini, au temps du vulgaire (si ce n'est au «temps vulgaire») qui est celui de nos blessures et de nos attentes anxieuses, c'est-à-dire de l'agitation fébrile et vaine des passions, et instant «tenu », stabilisé, contenant en lui-même la totalité du temps $\mathrm{d}^{\prime}$ une vie, et dans lequel vertu et bonheur ne font qu'un. Chez Heidegger, on pourrait même trouver la trace de cette distinction entre l'activité parfaite à chaque instant et prenant place dans le présent

\footnotetext{
${ }^{56}$ Ibid., p. 49 et 199.

${ }^{57}$ Ibid., p. 199. La première citation dans la citation est de Marc-Aurèle (Pensées, VIII, 32) ; la seconde, dont la provenance n'est pas indiquée, est bien sûr de Valéry (« Le cimetière marin »). 58 Marc-Aurèle, Pensées, XI, 1.
} 
délimité, et l'activité tournée vers un but extérieur qui demeure imparfaite - la distinction stoïcienne entre telos et skopos, la fin et le but d'une action : le telos du sage, sa fin, étant de faire tout ce qui est en son pouvoir pour atteindre son but (skopos), en sorte que, même en cas d'échec extérieur de son action, même dans le cas où son but $n$ 'est pas atteint, son action demeure aussi parfaite et irréprochable qu'auparavant. En effet, la résolution qui rassemble et unifie l'existence tout entière et permet d'atteindre au Dasein son être-un-tout dans le clin d'œil de l'instant est précisément de l'ordre d'un agir, mais d'un agir « originaire» qui est parfaitement achevé dans l'instant et n'attend pas des succès extérieurs sa récompense : "En étant résolu, le Dasein agit déjà $»^{59}$, écrit Heidegger; et il précise dans l'Introduction à la métaphysique:

Celui qui veut, celui qui place son Dasein tout entier dans un vouloir, est résolu (ist erschlossen). La résolution ne renvoie rien au lendemain, ne fait pas défaut, mais agit déjà dans l'instant et sans relâche. La résolution n'est pas seulement une décision d'agir, mais une amorce décisive de l'agir, laquelle précède tout agir et le traverse de part en part $^{60}$.

Non seulement le Dasein, dans l'être-pour-la-mort et la résolution devançante, atteint à sa totalité et à son être-un-tout, mais il atteint aussi, de ce fait même, à sa stabilité existentielle, laquelle contraste avec «l'instabilité distraite (das zerstreute Unverweilen) » et l'agitation du On ${ }^{61}$ - et, par voie de conséquence, à la fermeté (Standfestigkeit) de son existence ${ }^{62}$. C'est probablement là l'aspect le plus patent du "stoïcisme» de l'ontologie fondamentale, et celui qui a été le plus souvent relevé. La possession de soi-même du Dasein, son être en propre et sur le mode de l'appropriation à soi-même, cette manière d'être que Heidegger qualifie aussi d'ipséité ou d'être-soi (Selbstheit, Selbstsein) équivaut à une constance ou à une fermeté (Standfestigkeit)

\footnotetext{
${ }^{59}$ M. Heidegger, Sein und Zeit, op. cit., p. 300 ; trad. citée, p. 214.

${ }^{60}$ M. Heidegger, Einführung in die Metaphysik, GA 40, p. 23 ; traduction de G. Kahn (modifiée), Introduction à la métaphysique, Paris, Gallimard, « Tel», 1967, p. 23. Le possible arrière-plan stoïcien de cette affirmation n'en exclut pas un autre, augustinien : voir Confessions, VIII, VIIII, 20 : Ibi enim facultas ea, quae voluntas, et ipsum velle jam facere erat: "ici, en effet, la faculté de faire était identique à la volonté, et le "vouloir" lui-même était déjà le "faire" ".

${ }^{61}$ M. Heidegger, Sein und Zeit, op. cit., p. 347 ; trad. citée, p. 242-243 : «Sous l'effet du s'attendre à... sautillant, le présentifier est de plus en plus remis à lui-même. Il présentifie pour le présent. Se prenant ainsi dans ses propres rets, l'instabilité distraite (das zerstreute Unverweilen) devient agitation (Aufenthaltslosigkeit). Ce mode du présent est le contre-phénomène de l'instant ».

62 Bien sûr, cette stabilité peut aussi évoquer, par-delà la référence stoïcienne, une attitude existentielle proprement chrétienne, celle qu'Augustin exprime en les termes suivants : nec certior de te, sed stabilior in te esse cupiebam (Confessions, VIII, I, 1).
} 
de son existence, grâce à laquelle le Dasein se soustrait à l'instabilité et à la dispersion de l'existence déchue et se ressaisit lui-même en totalité dans la cohésion de ses possibles et sous l'horizon de la mort - auto-constance (Selbst-ständigkeit) et autonomie (Selbstständigkeit) qui contrastent avec l'hétéronomie foncière du On :

Le phénomène du pouvoir-être authentique ouvre le regard au maintien du Soi-même au sens de l'avoir-conquis-sa-tenue. Le maintien de soi-même au double sens de la solidité et de la constance [ou fermeté] (Standfestigkeit) est la contre-possibilité authentique de l'absence de maintien de l'échéance ir-résolue. Le maintien de soimême [ou autonomie : Selbständigkeit] ne signifie existentialement rien d'autre que la résolution devançante. La structure ontologique de celle-ci dévoile l'existentialité de l'être-soi-même (Selbstheit) du Soimême $(\text { Selbst })^{63}$.

Bien sûr, la totalisation de l'instant heideggérien demeure assez différente dans sa nature même de celle du présent stoïcien, puisque la densité de ce dernier était acquise au moyen d'un refus de tout ce qui pouvait placer l'âme (ou plutôt l'hegemonikon) hors d'elle-même, de tout ce qui pouvait transporter extatiquement l'individu vers son passé ou son avenir, de toutes les "projections », identifiées à autant $\mathrm{d}$ 'illusions et de pathologies de l'âme, auxquelles il était enclin à $\mathrm{s}^{\prime}$ abandonner ; cette totalisation est une manière de trouver l'éternité dans le temps et non point un retour du Dasein à sa propre finitude ; elle est «divinisation » et non assomption de sa condition finie. Aussi, la constance ou la fermeté ne peuvent signifier exactement la même chose ici et là - toute perspective de «salut » restant étrangère à l'esprit de l'Hauptwerk heideggérien. Il n'en reste pas moins que ces proximités sont manifestes entre stoïcisme et ontologie fondamentale et difficiles à attribuer à une simple rencontre fortuite.

Si l'on accepte l'idée de deux sources - très différentes - de la conception de la temporalité authentique développée par Sein und Zeit, une «transposition » phénoménologique de l'antithèse paulinienne entre deux modalités du "vivre » de la vie facticielle originaire en relation à la parousie, d'une part, et une reprise de motifs stoïciens relatifs à "l'instant totalisant », pour reprendre l'expression de Victor Goldschmidt, de l'autre; si l'on admet que, dans cette construction-description phénoménologique, le motif de l'être-pourla-mort en vient à se substituer silencieusement à l'espérance de la

${ }^{63}$ M. Heidegger, Sein und Zeit, op. cit., p. 322 ; trad. citée (modifiée), p. 227-228. 
parousie, modifiant de ce fait même totalement l'idée de « veille » et substituant à l'insécurité de l'existence chrétienne (si fortement soulignée par Luther), en tant qu'incertitude du salut, l'angoisse dans son sens spécifiquement heideggérien, mais une angoisse destinée à donner lieu à une appropriation de l'existence qui revêt des accents distinctivement stoïciens, on pourrait alors aller jusqu'à dire que la temporalité authentique de Sein und Zeit procède d'une «stoïcisation » implicite de certaines structures de la temporalité eschatologique paulinienne. Le caractère dérivé que reçoit la surprise dans un tel dispositif pourrait donc procéder pour une part à peu près égale du modèle des fidèles vivant dans les ténèbres et qui se laissent surprendre par la venue du Messie parce que leur existence est tout entière pour ce monde-ci et du modèle du sage stoïcien dont l'attention, concentrée sur l'instant présent, ne se laisse distraire ni prendre de court par aucun événement inopiné. 